Pacific Journal of Mathematics

ORTHOGONAL BASES ARE SCHAUDER BASES AND 


\title{
ORTHOGONAL BASES ARE SCHAUDER BASES AND A CHARACTERIZATION OF $\Phi$-ALGEBRAS
}

\author{
Sherif El-Helaly and Taqdir Husain
}

\begin{abstract}
It is shown that every orthogonal basis in a topological algebra is a Schauder basis. A new class of topological algebras called $\Phi$-algebras is introduced. They are characterized in terms of seminorms. Among other results, a necessary and sufficient condition for a locally convex $s$-algebra with an unconditional orthogonal basis to be an $A$-convex algebra is given.
\end{abstract}

0. Introduction. One of the interesting questions in the classical basis theory is whether every basis in a topological vector space of a certain type is a Schauder basis. Positive answers to this question have been established in the situations where the open mapping theorem holds, for instance, when the topological vector space is an $F$-space (Arsove [1]), in particular, a Banach space.

In the context of topological algebras, the second author and Watson showed that an orthogonal basis in a locally $m$-convex algebra (a setting where the open mapping theorem does not necessarily hold) is always a Schauder basis ([7], Proposition 3.1). Here we establish this result in its ultimate general setting; indeed, we show that every orthogonal basis in a topological algebra is a Schauder basis (Theorem 1.1).

Improving upon a result of Husain and Watson [7], we show that every locally $m$-convex algebra with an identity and an orthogonal basis is topologically isomorphic with a dense subalgebra of the algebra $s$ of all complex sequences with the pointwise operations and the topology of pointwise convergence (Theorem 2.1).

In $\S 3$, we introduce a class of topological algebras with orthogonal basis which we call $\Phi$-algebras. Examples of such algebras are given and a characterization of $\Phi$-algebras in terms of seminorms is proved (Theorem 3.3).

In $\S 4$, we study different types of topological algebras with orthogonal bases. Among other results, we give a necessary and sufficient 
condition for a locally convex $s$-algebra with an unconditional orthogonal basis to be $A$-convex (Theorem 4.6); and we use this result to characterize the algebra $s$ (Theorem 4.7).

In the sequel, all topological algebras and topological vector spaces are Hausdorff with jointly continuous multiplication and over the field C.

For standard terms used here we refer the reader to Singer [11] and Zelazko [12]. In addition, we shall use the following definitions and results given in our paper [5]. Let $A$ be a topological algebra. A subset $S$ of $A$ is called squarely idempotent if for every $x, y \in A$ with $x^{2}, y^{2} \in S$ we have $x y \in S$. A seminorm $p$ on $A$ is called squarely submultiplicative if $p(x y) \leq \sqrt{p\left(x^{2}\right)} \sqrt{p\left(y^{2}\right)}$ for all, $x, y \in A$. A topological algebra with a base of squarely idempotent neighborhoods of 0 is called an s-algebra. It turns out that a locally convex algebra is an $s$-algebra if and only if it has a defining family of squarely submultiplicative seminorms ([5], Proposition 2.3). The significance of $s$-algebras lies in the following. An orthogonal basis in a Banach algebra need not be unconditional. However, an orthogonal basis in a $B_{0}$-algebra $A$ is unconditional if and only if $A$ is an $s$-algebra ([5], Examples 2.1 and Theorem 2.5).

1. Orthogonal bases are Schauder bases. A sequence $\left\{e_{n}\right\}$ in a topological vector space $E$ is said to form a basis in $E$ if for each $x \in E$ there exists a unique sequence $\left\{e_{n}^{*}(x)\right\}$ of scalars such that $x=$ $\sum_{n=1}^{\infty} e_{n}^{*}(x) e_{n}$. Each $e_{n}^{*}$ is a linear functional. If each $e_{n}^{*}$ is continuous, $\left\{e_{n}\right\}$ is called a Schauder basis. A basis need not be a Schauder basis. However, if $E$ is an $F$-space [1] or, in particular, a Banach space, then by the open mapping theorem it follows that every basis in $E$ is a Schauder basis.

If $A$ is a topological algebra, then a basis in $A$, as a topological vector space, is called orthogonal provided that $e_{m} e_{n}=\delta_{m n} e_{n}$ for all $m, n \in \mathbf{N}$, where $\delta_{m n}$ is the Kronecker delta. An orthogonal basis in a topological algebra is unique up to a permutation, if it exists [7].

Husain and Watson [7] showed that every orthogonal basis in a locally $m$-convex algebra is a Schauder basis. Here we show (Theorem 1.1) that this is true in general for any topological algebra.

1.1. Theorem. An orthogonal basis $\left\{e_{n}\right\}$ in a topological algebra $A$ is a Schauder basis. 
Proof. Let $n \in \mathbf{N}$. For each $x \in A, x=\sum_{m=1}^{\infty} e_{m}^{*}(x) e_{m}$ implies

$$
e_{n} x=e_{n}^{*}(x) e_{n} \text {. }
$$

Since $e_{n} \neq 0$ and $A$ is Hausdorff, there exists a circled neighborhood $U$ of $0 \in A$ such that $e_{n} \notin U$. From the continuity of multiplication, we find a circled neighborhood $V$ of 0 with $V V \subseteq U$. Put

$$
r=\inf \left\{d>0: e_{n} \in d U\right\} .
$$

Since $U$ is circled and $e_{n} \notin U$, we have $r \geq 1>0$. Since each neighborhood of 0 is absorbing, we find $t>0$ with $e_{n} \in t V$. Put

$$
W=\left(t^{-1} r\right) V \text {. }
$$

To complete the proof, we show that $\left|e_{n}^{*}(x)\right| \leq 1$ for all $x \in W$. Indeed for $x \in W$,

$$
e_{n}^{*}(x) r^{-1} e_{n}=r^{-1} x e_{n}=t r^{-1} x \cdot t^{-1} e_{n} \in V \subseteq U .
$$

Since $U$ is circled, we have $\left|e_{n}^{*}(x)\right| r^{-1} e_{n} \in U$. If $e_{n}^{*}(x) \neq 0$, then $e_{n} \in\left|e_{n}^{*}(x)\right|^{-1} r U$. Hence, by the definition of $r$, we have $\left|e_{n}^{*}(x)\right|^{-1} r \geq r$ and consequently $\left|e_{n}^{*}(x)\right| \leq 1$, as required.

1.2. Notations. Let $A$ be a topological algebra with an orthogonal basis $\left\{e_{n}\right\}$. Each $x \in A$ can be expressed as $x=\sum_{n=1}^{\infty} e_{n}^{*}(x) e_{n}$. Let $\hat{x}$ denote the element of $s$ given by $\hat{x}(n)=e_{n}^{*}(x), n \in \mathbf{N}$. The set $\hat{A}=\{\hat{x}: x \in A\}$ constitutes a subalgebra of $s$ and $\sigma: x \rightarrow \hat{x}$ is an algebra isomorphism of $A$ onto $\hat{A}$. We endow $\hat{A}$ with the pointwise convergence topology induced from $s$.

1.3. Corollary. Let $A$ be a topological algebra with an orthogonal basis $\left\{e_{n}\right\}$. Then

(i) $\hat{A}$ is dense in $s$ and the isomorphism $\sigma$ is continuous.

(ii) A nonzero multiplicative linear functional $f$ on $A$ is continuous if and only if $f=e_{k}^{*}$ for some $k \in \mathbf{N}$.

Proof. (i) The continuity of $\sigma$ is a consequence of the continuity of each $e_{n}^{*}$ (Theorem 1.1). Since $\hat{A}$ clearly contains all sequences of scalars with finitely many nonzero terms, it follows that $\hat{A}$ is dense in S.

(ii) If $f=e_{n}^{*}$ for some $n \in \mathbf{N}$, then, by Theorem 1.1, $f$ is continuous. To prove the converse, we notice that

$$
f\left(e_{n}\right) f(x)=f\left(e_{n} x\right)=f\left(e_{n}^{*}(x) e_{n}\right)=e_{n}^{*}(x) f\left(e_{n}\right)
$$


for every $x \in A$ and $n \in \mathbf{N}$. Since $f$ is continuous and nonzero, we have $f\left(e_{k}\right) \neq 0$ for some $k \in \mathbf{N}$. It follows that $f=e_{k}^{*}$.

2. Locally $m$-convex algebras with orthogonal bases. Let $A$ be a complete locally $m$-convex algebra with an orthogonal basis and an identity. Husain and Watson [7] showed that $A$ is topologically isomorphic with the algebra $s$. Here, with the assumption of completeness dropped, we show that $A$ is topologically isomorphic with a dense subalgebra of $s$ (Theorem 2.1).

2.1. Theorem. Let $A$ be a locally $m$-convex algebra with an orthogonal basis $\left\{e_{n}\right\}$ and let $P$ be a defining family of submultiplicative seminorms. Consider the following statements:

(i) $\sigma: A \rightarrow s$ is onto.

(ii) $A$ has an identity.

(iii) For each $p \in P, p\left(e_{n}\right) \neq 0$ for at most finitely many $e_{n}$ 's.

(iv) $A$ is topologically isomorphic with a dense subalgebra of $s$.

(v) $A$ is topologically isomorphic with $s$.

Then (i) $\Rightarrow$ (ii) $\Rightarrow$ (iii) $\Rightarrow$ (iv). If $A$ is complete, then all the five statements are equivalent.

Proof. For the first two implications, see Lemma 3.2 of [7]. Under condition (iii), the topology generated by $P$ on $\hat{A}$ via the isomorphism $\sigma$ is the same as the topology induced from $s$. Hence, by Corollary 1.3 (i) we have (iii) $\Rightarrow$ (iv). Finally, it is clear that (v) $\Rightarrow$ (i) and under the additional hypothesis of completeness of $A$ we have (iv) $\Rightarrow(\mathrm{v})$.

From Corollary 1.3 (i) we see that a topological algebra $A$ with an orthogonal basis has a continuous isomorphic image in $s$. Further, if $A$ is locally $m$-convex, this continuous image becomes a homeomorphic one when $A$ has an identity (Theorem 2.1).

2.2. Corollary. Let $A$ be a topological algebra with an orthogonal basis $\left\{e_{n}\right\}$ and an identity $e$. There is one and only one locally $m$ convex topology on $A$ coarser than its original topology, namely, the relative pointwise convergence topology on $\hat{A}$ transferred to $A$ via $\sigma$.

Proof. Let $\tau$ be a locally $m$-convex topology on $A$ coarser than its original topology. For each $x \in A$, the convergence of $\sum_{n=1}^{\infty} e_{n}^{*}(x) e_{n}$ to $x$ for the original topology implies its convergence to $x$ for the coarser topology $\tau$ and so $\left\{e_{n}\right\}$ remains an orthogonal basis in $A$ when $A$ is 
endowed with $\tau$. The proof is completed by an appeal to Theorem 2.1 and Corollary 1.3 (i).

3. $\Phi$-algebras. Let $A$ be a topological algebra with an orthogonal basis. As we have seen in $\S 2, \sigma: A \rightarrow \hat{A}$ is a continuous isomorphism when $\hat{A}$ is given the relative pointwise convergence topology induced from $s$. This topology is generated by the family of seminorms

$$
p_{\varphi}(\hat{x})=\sup \{|\varphi(n) \hat{x}(n)|: n \in \mathbf{N}\},
$$

$\hat{x} \in \hat{A}$, where $\varphi$ ranges over the set $C_{00}^{+}(\mathbf{N})$ of all nonnegative functions on $\mathbf{N}$ with finite support. This motivates the following

3.1. Definition. A topological algebra $A$ with an orthogonal basis $\left\{e_{n}\right\}$ is called a $\Phi$-algebra if a family $\Phi$ of nonnegative functions on $\mathbf{N}$ generates a topology on $\hat{A}$ by means of seminorms

$$
p_{\varphi}(\hat{x})=\sup \{|\varphi(n) \hat{x}(n)|: n \in \mathbf{N}\} \text {, }
$$

$\hat{x} \in \hat{A}, \varphi \in \Phi$, such that $\sigma: A \rightarrow\left(\hat{A},\left\{p_{\varphi}: \varphi \in \Phi\right\}\right)$ is a topological isomorphism.

In addition to the algebra $s$, we have the following two examples of $\Phi$-algebras.

3.2. EXAmples. (i) Let $C_{b}(\mathbf{N})$ denote the algebra of all bounded complex sequences with the pointwise operations and let $C_{0}^{+}(\mathbf{N})$ be the set of all nonnegative sequences converging to zero. The strict topology [2] on $C_{b}(\mathbf{N})$ is defined by the seminorms

$$
p_{\varphi}(x)=\sup \{|\varphi(n) x(n)|: n \in \mathbf{N}\},
$$

$x \in C_{b}(\mathbf{N}), \varphi \in C_{0}^{+}(\mathbf{N})$. With this topology, $C_{b}(\mathbf{N})$ is a complete $A$ convex [3] algebra with identity. The sequence $\left\{e_{n}\right\}$, where $e_{n}(m)=$ $\delta_{m n}$ is an orthogonal basis in $C_{b}(\mathbf{N})$. Clearly, $C_{b}(\mathbf{N})$ is a $\Phi$-algebra with $\Phi=C_{0}^{+}(\mathbf{N})$. Since $C_{b}(\mathbf{N})$ is not topologically isomorphic with $s$, it follows from Theorem 2.1 that $C_{b}(\mathbf{N})$ is not locally $m$-convex.

(ii) Let $\mathbf{D}$ be the open unit disc in the complex plane and let $H(\mathbf{D})$ denote the Fréchet space of all analytic functions on $\mathbf{D}$ with the pointwise addition and scalar multiplication, and the compact-open topology. For each $n \in\{0\} \cup \mathbf{N}$ let $e_{n}$ be the element in $H(\mathbf{D})$ given by $e_{n}(z)=z^{n}, z \in \mathbf{D}$. Then for each $f \in H(\mathbf{D})$ we have $f=\sum_{n=0}^{\infty} e_{n}^{*}(f) e_{n}$ converging in $H(\mathbf{D})$, with $e_{n}^{*}(f)$ being the $n$th Taylor coefficient of $f$. 
This shows that $\left\{e_{n}\right\}$ is a basis in $H(\mathbf{D})$. Endowed with the Hadamard multiplication

$$
(f g)(z)=(2 \pi i)^{-1} \int_{|u|=r} f(u) g\left(z u^{-1}\right) u^{-1} d u,
$$

$|z|<r<1 ; H(\mathbf{D})$ is a $B_{0}$-algebra [10]. The $n$th Taylor coefficient of $f g$ is $e_{n}^{*}(f) e_{n}^{*}(g)[\mathbf{1 0}]$ and hence,

$$
f g=\sum_{n=0}^{\infty} e_{n}^{*}(f) e_{n}^{*}(g) e_{n}
$$

which shows that the basis $\left\{e_{n}\right\}$ is orthogonal in $H(\mathbf{D})$. The element $e \in H(\mathbf{D})$ defined by $e(z)=(1-z)^{-1}=\sum_{n=0}^{\infty} z^{n}$ is an identity in $H(\mathbf{D})$.

An equivalent family of seminorms on $H(\mathbf{D})$ is given by

$$
\|f\|_{r}=\sup \left\{\left|e_{n}^{*}(f) r^{n}\right|: n \in \mathbf{N}\right\} \text {, }
$$

$0<r<1$ (cf. [8], p. 45). This shows that $H(\mathbf{D})$ is a $\Phi$-algebra with $\Phi=\left\{\varphi_{r}: 0<r<1\right\}$ where $\varphi_{r}(n)=r^{n}, n \in\{0\} \cup \mathbf{N}$. By Theorem 2.1, $H(\mathbf{D})$ is not locally $m$-convex. Since every barrelled $A$-convex algebra is locally $m$-convex [9], $H(\mathbf{D})$ is not $A$-convex either.

Now we have a characterization of $\Phi$-algebras.

3.3. THEOREM. Let $A$ be a locally convex algebra with an orthogonal basis $\left\{e_{n}\right\}$ and an identity $e$. Then $A$ is a $\Phi$-algebra if and only if $a$ defining family $P$ of seminorms on $A$ satisfies the following condition

(*): For all $x, y \in A$ and $p, q \in P$,

$$
p\left(e_{n}^{*}(x) e_{n}\right) \leq q\left(e_{n}^{*}(y) e_{n}\right) \quad \text { for all } n \in \mathbf{N} \text { implies } p(x) \leq q(y) .
$$

Proof. $(\Rightarrow)$ : It is clear that the seminorms described in Definition 3.1 satisfy condition $(*)$.

$(\Leftarrow)$ : For each $p \in P, n \in \mathbf{N}$ and $x \in A$ put $\varphi_{p}(n)=p\left(e_{n}\right)$. We then have

$$
\varphi_{p}(n) \hat{x}(n)=p\left(e_{n}\right) e_{n}^{*}(x)=p\left(e_{n}^{*}(x) e_{n}\right)
$$

and from the convergence of $\sum_{n=1}^{\infty} e_{n}^{*}(x) e_{n}$ it follows that

$$
\sup \left\{\left|\varphi_{p}(n) \hat{x}(n)\right|: n \in \mathbf{N}\right\}<\infty \text {. }
$$

Set

$$
\|\hat{x}\|_{p}=\sup \left\{\left|\varphi_{p}(n) \hat{x}(n)\right|: n \in \mathbf{N}\right\} .
$$

Clearly, $\left\{\|\cdot\|_{p}: p \in P\right\}$ is a family of seminorms on $\hat{A}$. We show that $\sigma: A \rightarrow\left(\hat{A},\left\{\|\cdot\|_{p}: p \in P\right\}\right)$ is a homeomorphism, which will complete the proof. 
Let $x \in A, m \in \mathbf{N}$ and $p \in P$; and put $x_{m}=e_{m}^{*}(x) e_{m}$. Clearly, $p\left(e_{n}^{*}\left(x_{m}\right) e_{n}\right)=\delta_{m n} p\left(e_{m}^{*}(x) e_{m}\right)$ for all $n \in \mathbf{N}$ and hence by condition $(*)$ we have $p\left(x_{m}\right) \leq p(x)$. It follows that

$$
\|\hat{x}\|_{p}=\sup \left\{p\left(x_{m}\right): m \in \mathbf{N}\right\} \leq p(x) .
$$

This shows that $\sigma: A \rightarrow\left(\hat{A},\left\{\|\cdot\|_{p}: p \in P\right\}\right)$ is continuous; and it remains to show that it is open.

Let $p \in P$. Find $q \in P$ such that $p(x y) \leq q(x) q(y)$ for all $x, y \in A$. To complete the proof we show that there exists $K>0$ such that $p(x) \leq K$ for all $x \in A$ with $\|\hat{x}\|_{q} \leq 1$. Indeed, for such $x$ and for every $n \in \mathbf{N}$ we have

$$
\begin{aligned}
p\left(e_{n}^{*}(x) e_{n}\right) & =\left|e_{n}^{*}(x) p\left(e_{n}\right)\right|=\left|e_{n}^{*}(x) p\left(e_{n}^{2}\right)\right| \\
& \leq\left|e_{n}^{*}(x)\right|\left(q\left(e_{n}\right)\right)^{2} \leq\|\hat{x}\|_{q} q\left(e_{n}\right) \leq q\left(e_{n}\right)=q\left(e_{n}^{*}(e) e_{n}\right),
\end{aligned}
$$

where the rightmost equality follows because $e_{n}^{*}(e)=1$. Thus from condition (*) we have $p(x) \leq q(e)$. We also have $q(e) \neq 0$ for otherwise $p(x)=p(x e) \leq q(x) q(e)=0$ for all $x \in A$. Take $K=q(e)$.

3.4. REMARK. The Banach algebra $\ell_{1}$ with the pointwise operations has the orthogonal basis $\left\{e_{n}\right\}$ where $e_{n}(m)=\delta_{m n}$. It is easy to verify that the $\ell_{1}$ norm satisfies condition $(*)$ of Theorem 3.3 and that, on the other hand, $\ell_{1}$ is not a $\Phi$-algebra. Note that $\ell_{1}$ does not have an identity. We do not know an example of a locally convex algebra with an orthogonal basis and an identity which is not a $\Phi$-algebra.

4. $A$-convex algebras and locally convex $s$-algebras with orthogonal bases. In our paper [5] we introduced and studied $s$-algebras (see the introduction of the present paper for the definition). It is easy to verify that every $\Phi$-algebra is an $s$-algebra. The converse is not true; indeed, the Banach algebra $\ell_{1}$ is an $s$-algebra [5] which is not a $\Phi$-algebra (Remark 3.4). Further, for $1 \leq p<\infty$, the convolution algebra $L_{p}(\mathbf{T})$ over the torus group $\mathbf{T}$ is a Banach algebra which is not an $s$-algebra [5]; and $H(\mathbf{D})$ (Example 3.2 (ii)) is an $s$-algebra which is not $A$-convex. Theorem 4.6 below gives a necessary and sufficient condition for a locally convex $s$-algebra with an unconditional orthogonal basis to be $A$-convex; and Theorem 4.7 characterizes the algebra $s$. For further information about $s$-algebras see [5].

First we have Theorem 4.1 and Corollary 4.2 the proofs of which are omitted since the proofs of these results known for Banach spaces ([11], Theorem 16.1) can be adapted for the more general case of locally convex spaces. (Detailed proofs for the locally convex space case are given in [4], Theorem III.17 and Corollary III.18.) 
4.1. THEOREM. Let $E$ be a locally convex space with an unconditional basis $\left\{e_{n}\right\}$. Let $u$ be the topology of $E$ defined by a family $\left\{\|\cdot\|_{h}: h \in H\right\}$ of seminorms. For $x \in E$ and $h \in H$ put

$$
\|x\|_{h}^{\prime}=\sup \left\{\sum_{n=1}^{\infty}\left|e_{n}^{*}(x)\right|\left|f\left(e_{n}\right)\right|: f \in F_{h}\right\}
$$

and

$$
\|x\|_{h}^{\prime \prime}=\sup \left\{\left\|\sum_{n \in J} e_{n}^{*}(x) e_{n}\right\|_{h}: J \in \beta\right\}
$$

where $F_{h}$ is the set of all linear functionals on $E$ with $|f(x)| \leq\|x\|_{h}$ for all $x \in E$; and $\beta$ is the set of all finite subsets of $\mathbf{N}$. Then each of $\left\{\|\cdot\|_{h}^{\prime}: h \in H\right\}$ and $\left\{\|\cdot\|_{h}^{\prime \prime}: h \in H\right\}$ is a family of seminorms defining topologies $u^{\prime}$ and $u^{\prime \prime}$, respectively, on $E$ such that $\|x\|_{h} \leq\|x\|_{h}^{\prime \prime} \leq\|x\|_{h}^{\prime}$ for all $x \in E$ and $h \in H$. Moreover, $u \subseteq u^{\prime}=u^{\prime \prime}$.

4.2. Corollary. If $E$ in Theorem 4.1 is a Fréchet space, then $u=$ $u^{\prime}=u^{\prime \prime}$ and $\left\{e_{n}\right\}$ is a Schauder basis.

We note that the conclusion of Corollary 4.2 follows from the open mapping theorem which is valid in Fréchet spaces. In the topological algebra setting we prove the same conclusion under different conditions.

4.3. Corollary. Let $A$ be a locally convex s-algebra with an unconditional orthogonal basis $\left\{e_{n}\right\}$. Let $u$ be the topology of $A$ defined by a family $\left\{\|\cdot\|_{h}: h \in H\right\}$ of squarely submultiplicative seminorms. With the same notations as in Theorem 4.1 we have $u=u^{\prime}=u^{\prime \prime}$. (Note that $\left\{e_{n}\right\}$ is already a Schauder basis by Theorem 1.1.)

Proof. In view of Theorem 4.1, it is enough to show that $u^{\prime \prime} \subseteq u$. Let $x \in A, h \in H$ and let $\varepsilon>0$ be given. Since the basis $\left\{e_{n}\right\}$ is unconditional, the net $\left\{\sum_{n \in J} e_{n}^{*}(x) e_{n}\right\}_{J \in \beta}$ (where $\beta$ is the set of all finite subsets of $\mathbf{N}$ ordered by inclusion) converges to $x$. Hence, there exists $J_{0} \in \beta$ such that for $J \in \beta$ with $J_{0} \subseteq J$ we have

$$
\left\|\sum_{n \in J} e_{n}^{*}(x) e_{n}\right\|_{h}<\|x\|_{h}+\varepsilon .
$$

Let $I \in \beta$. Then by the orthogonality of the basis $\left\{e_{n}\right\}$ we have

$$
\sum_{n \in I} e_{n}^{*}(x) e_{n}=\sum_{n \in I} e_{n}^{*}(x) e_{n} \cdot \sum_{n \in I \cup J_{0}} e_{n}^{*}(x) e_{n}
$$


and hence

$$
\left(\left\|\sum_{n \in I} e_{n}^{*}(x) e_{n}\right\|_{h}\right)^{2} \leq\left\|\sum_{n \in I} e_{n}^{*}(x) e_{n}\right\|_{h} \cdot\left\|\sum_{n \in I \cup J_{0}} e_{n}^{*}(x) e_{n}\right\|_{h},
$$

since $\|\cdot\|_{h}$ is squarely submultiplicative. Consequently,

$$
\left\|\sum_{n \in I} e_{n}^{*}(x) e_{n}\right\|_{h} \leq\left\|\sum_{n \in I \cup J_{0}} e_{n}^{*}(x) e_{n}\right\|_{h}<\|x\|_{h}+\varepsilon
$$

for all $I \in \beta$. Hence, from the definition of $\|\cdot\|_{h}^{\prime \prime}$ we have

$$
\|x\|_{h}^{\prime \prime}<\|x\|_{h}+\varepsilon
$$

Since $\varepsilon>0$ is arbitrary, we have $\|x\|_{h}^{\prime \prime} \leq\|x\|_{h}$.

4.4. Proposition. Let $A$ be an A-convex algebra with an orthogonal basis $\left\{e_{n}\right\}$ and let $P$ be an absorbing family of seminorms defining the topology of $A$. Then for each $x \in A$ and $p \in P$, the set $\left\{e_{n}^{*}(x): n \in\right.$ $\left.\mathbf{N}, p\left(e_{n}\right) \neq 0\right\}$ is bounded.

Proof. It follows from the definition of $A$-convexity that for each $x \in A$ and $p \in P$ there exists $K(p, x)>0$ such that $p(x y) \leq$ $K(p, x) p(y)$ for all $y \in A$. In particular,

$$
\left|e_{n}^{*}(x)\right| p\left(e_{n}\right)=p\left(e_{n}^{*}(x) e_{n}\right)=p\left(x e_{n}\right) \leq K(p, x) p\left(e_{n}\right)
$$

for all $n \in \mathbf{N}$. We then have $\left|e_{n}^{*}(x)\right| \leq K(p, x)$ for all $n \in \mathbf{N}$ with $p\left(e_{n}\right) \neq 0$.

4.5. Corollary. If, in addition to the hypothesis of Proposition 4.4, there exists $p \in P$ with $P\left(e_{n}\right) \neq 0$ for all $n \in \mathbf{N}$, then for each $x \in A$, the set $\left\{e_{n}^{*}(x): n \in \mathbf{N}\right\}$ is bounded.

4.6. TheOREM. Let $A$ be a locally convex s-algebra with an unconditional orthogonal basis $\left\{e_{n}\right\}$. Then $A$ is $A$-convex if and only if $A$ has a defining family $P$ of seminorms such that for each $x \in A$ and $p \in P$, the set $\left\{e_{n}^{*}(x): n \in \mathbf{N}, p\left(e_{n}\right) \neq 0\right\}$ is bounded.

Proof. The "only if" part follows from Proposition 4.4. For the "if" part, assume that $A$ has a defining family of seminorms satisfying the stated condition. It follows that the same condition is satisfied by every defining family of seminorms, as can easily be verified. In particular, the condition is satisfied by a defining family $\left\{\|\cdot\|_{h}: h \in H\right\}$ 
of squarely submultiplicative seminorms, which exists because $A$ is a locally convex $s$-algebra. For each $x \in A$ and $h \in H$ there exists $K(h, x)>0$ such that $\left|e_{n}^{*}(x)\right| \leq K(h, x)$ for all $n \in T_{h}$, where $T_{h}=$ $\left\{n \in \mathbf{N}:\left\|e_{n}\right\|_{h} \neq 0\right\}$. From Corollary 4.3 with the same notations we have $u=u^{\prime}$. We complete the proof by showing that each $\|\cdot\|_{h}^{\prime}, h \in H$ is absorbing. For $f \in F_{h}$ (as defined in Theorem 4.1) and $n \in \mathbf{N} \backslash T_{h}$ we have $\left|f\left(e_{n}\right)\right| \leq\left\|e_{n}\right\|_{h}=0$ and hence for $x \in A$,

$$
\begin{aligned}
\|x y\|_{h}^{\prime} & =\sup \left\{\sum_{n \in \mathbf{N}}\left|e_{n}^{*}(x)\right|\left|e_{n}^{*}(y)\right|\left|f\left(e_{n}\right)\right|: f \in F_{h}\right\} \\
& =\sup \left\{\sum_{n \in T_{h}}\left|e_{n}^{*}(x)\right|\left|e_{n}^{*}(y)\right|\left|f\left(e_{n}\right)\right|: f \in F_{h}\right\} \\
& \leq K(h, x) \sup \left\{\sum_{n \in T_{h}}\left|e_{n}^{*}(y)\right|\left|f\left(e_{n}\right)\right|: f \in F_{h}\right\} \\
& =K(h, x) \sup \left\{\sum_{n \in \mathbf{N}}\left|e_{n}^{*}(y)\right|\left|f\left(e_{n}\right)\right|: f \in F_{h}\right\} \\
& =K(h, x)\|y\|_{h}^{\prime}
\end{aligned}
$$

for every $y \in A$ and so $\|\cdot\|_{h}^{\prime}$ is absorbing.

4.7. THEOREM. Let $A$ be a $B_{0}$-algebra with an unconditional orthogonal basis $\left\{e_{n}\right\}$ and an identity $e$. Then $A$ is topologically isomorphic with the algebra $s$ if and only if $A$ has a defining family $P$ of seminorms such that for all $x \in A$ and $p \in P$ the set $\left\{e_{n}^{*}(x): n \in \mathbf{N}, p\left(e_{n}\right) \neq 0\right\}$ is bounded.

Proof. If $A$ is topologically isomorphic with $s$ then, by Theorem 2.1, $A$ has a defining family $P$ of seminorms such that the set $\{n \in$ $\left.\mathbf{N}: p\left(e_{n}\right) \neq 0\right\}$ is finite for every $p \in P$. It is clear that the family $P$ satisfies the stated condition.

To prove the converse, notice that $A$ is an $s$-algebra because it is a $B_{0}$-algebra with an unconditional orthogonal basis ([5], Theorem 2.5). Thus, if $A$ has a defining family $P$ of seminorms satisfying the stated condition, then $A$ is $A$-convex by Theorem 4.6. Now, being $A$-convex and barrelled, $A$ is locally $m$-convex [9]. The proof is completed by an appeal to Theorem 2.1.

In Example 3.2 (ii) above we showed that $H(\mathrm{D})$ is not $A$-convex. This also follows from Theorem 4.6 by considering the equivalent 
family $\left\{\|\cdot\|_{r}: 0<r<1\right\}$ of seminorms (given in Example 3.2 (ii)), together with any element in $H(\mathrm{D})$ with an unbounded set of Taylor coefficients.

\section{REFERENCES}

[1] M. G. Arsove, The Paley-Wiener theorem in metric linear spaces, Pacific J. Math., 10 (1960), 365-379.

[2] R. C. Buck, Bounded continuous functions on a locally compact space, Michigan Math. J., 5 (1958), 95-104.

[3] A. Cochran, Topological algebras and Mackey topologies, Proc. Amer. Math. Soc., 30 (1971), 115-119.

[4] S. El-Helaly, Topological algebras with orthogonal M-bases, Ph.D. Thesis, McMaster University, Hamilton, Ontario, 1985.

[5] S. El-Helaly and T. Husain, Unconditionality of orthogonal bases in $B_{0}$-algebras, Commentationes Mathematicae, (to appear).

[6] T. Husain and J. Liang, Multiplicative functionals on Fréchet algebras with bases, Canad. J. Math., XXIX (1977), 270-276.

[7] T. Husain and S. Watson, Topological algebras with orthogonal Schauder bases, Pacific J. Math., 91 (1980), 339-347.

[8] D. H. Luecking and L. A. Rubel, Complex Analysis: A Functional Analytic Approach, Springer Verlag, New York, Berlin, Heidelberg, Tokyo, 1984.

[9] E. Michael, Locally multiplicatively convex topological algebras, Amer. Math. Soc. Memoirs \#11, 1952.

[10] R. Porcelli, Linear Spaces of Analytic Functions, Rand McNally and Company, Chicago, 1966.

[11] I. Singer, Bases in Banach Spaces I, Springer-Verlag, New York, Berlin, Heidelberg, 1970.

[12] W. Zelazko, Banach Algebras, Elsevier, Amsterdam, 1973.

Received November 29, 1986 and in revised form July 20, 1987. The work of the second author was partially supported by an NSERC grant.

Pennsylvania State University

WORTHINGTON SCRANTON CAMPUS

DUNMORE, PA 18512 U.S.A

AND

MCMASTER UNIVERSITY

Hamilton, Ontario, L8S 4K1 Canada 



\title{
PACIFIC JOURNAL OF MATHEMATICS EDITORS
}

\author{
V. S. VARADARAJAN \\ (Managing Editor) \\ University of California \\ Los Angeles, CA 90024 \\ HERBERT Clemens \\ University of Utah \\ Salt Lake City, UT 84112 \\ R. FINN \\ Stanford University \\ Stanford, CA 94305
}

\author{
HERMANN FLASCHKA \\ University of Arizona \\ Tucson, AZ 85721
}

Ramesh A. Gangolli University of Washington Seattle, WA 98195

VAUGHAN F. R. JONES University of California Berkeley, CA 94720
ROBION KIRBY

University of California

Berkeley, CA 94720

C. C. MOORE

University of California

Berkeley, CA 94720

HAROLD STARK

University of California, San Diego La Jolla, CA 92093

\section{ASSOCIATE EDITORS}

\author{
R. ARENS \\ E. F. BECKENBACH \\ B. H. NEUMANN \\ F. WOLF \\ K. YOSHIDA \\ (1906-1982)

\section{SUPPORTING INSTITUTIONS}

\section{UNIVERSITY OF ARIZONA} \\ UNIVERSITY OF BRITISH COLUMBIA \\ CALIFORNIA INSTITUTE OF TECHNOLOGY \\ UNIVERSITY OF CALIFORNIA \\ MONTANA STATE UNIVERSITY \\ UNIVERSITY OF NEVADA, RENO \\ NEW MEXICO STATE UNIVERSITY \\ OREGON STATE UNIVERSITY \\ UNIVERSITY OF OREGON \\ UNIVERSITY OF SOUTHERN CALIFORNIA \\ STANFORD UNIVERSITY \\ UNIVERSITY OF HAWAII \\ UNIVERSITY OF TOKYO \\ UNIVERSITY OF UTAH \\ WASHINGTON STATE UNIVERSITY \\ UNIVERSITY OF WASHINGTON
}

The Supporting Institutions listed above contribute to the cost of publication of this Journal, but they are not owners or publishers and have no responsibility for its content or policies.

Mathematical papers intended for publication in the Pacific Journal of Mathematics should be in typed form or offset-reproduced (not dittoed), double spaced with large margins. Please do not use built up fractions in the text of the manuscript. However, you may use them in the displayed equations. Underline Greek letters in red, German in green, and script in blue. The first paragraph must be capable of being used separately as a synopsis of the entire paper. In particular it should contain no bibliographic references. Please propose a heading for the odd numbered pages of less than 35 characters. Manuscripts, in triplicate, may be sent to any one of the editors. Please classify according to the scheme of Math. Reviews, Index to Vol. 39. Supply name and address of author to whom proofs should be sent. All other communications should be addressed to the managing editor, or Elaine Barth, University of California, Los Angeles, California 90024

There are page-charges associated with articles appearing in the Pacific Journal of Mathematics. These charges are expected to be paid by the author's University, Government Agency or Company. If the author or authors do not have access to such Institutional support these charges are waived. Single authors will receive 50 free reprints; joint authors will receive a total of 100 free reprints. Additional copies may be obtained at cost in multiples of 50 .

The Pacific Journal of Mathematics is issued monthly as of January 1966. Regular subscription rate: $\$ 190.00$ a year (5 Vols., 10 issues). Special rate: $\$ 95.00$ a year to individual members of supporting institutions.

Subscriptions, orders for numbers issued in the last three calendar years, and changes of address should be sent to Pacific Journal of Mathematics, P.O. Box 969, Carmel Valley, CA 93924, U.S.A. Old back numbers obtainable from Kraus Periodicals Co., Route 100, Millwood, NY 10546.

The Pacific Journal of Mathematics at P.O. Box 969, Carmel Valley, CA 93924 (ISSN 0030-8730) publishes 5 volumes per year. Application to mail at Second-class postage rates is pending at Carmel Valley, California, and additional mailing offices. Postmaster: send address changes to Pacific Journal of Mathematics, P.O. Box 969, Carmel Valley, CA 93924.

PUBLISHED BY PACIFIC JOURNAL OF MATHEMATICS, A NON-PROFIT CORPORATION Copyright (C) 1988 by Pacific Journal of Mathematics 


\section{Pacific Journal of Mathematics}

\section{Vol. 132, No. $2 \quad$ February, 1988}

Jeffery Marc Bergen and Luisa Carini, A note on derivations with power central values on a Lie ideal ..............................209

Alfonso Castro and Sumalee Unsurangsie, A semilinear wave equation

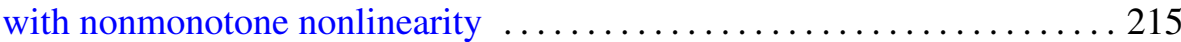

Marius Dadarlat, On homomorphisms of matrix algebras of continuous

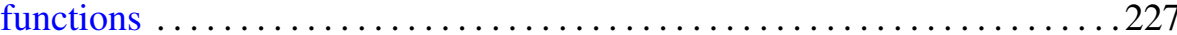

A. Didierjean, Quelques classes de cobordisme non orienté refusant de se

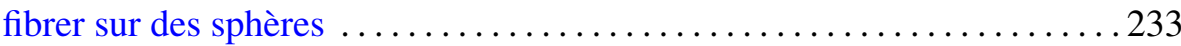

Edward George Effros and Zhong-Jin Ruan, On matricially normed spaces

Sherif El-Helaly and Taqdir Husain, Orthogonal bases are Schauder bases

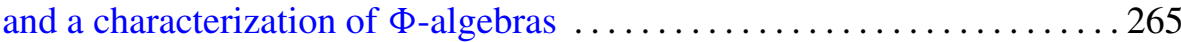

Edward Richard Fadell and Peter N-S Wong, On deforming $G$-maps to be

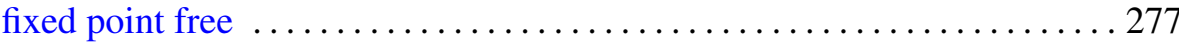

Jean-Jacques Gervais, Stability of unfoldings in the context of equivariant

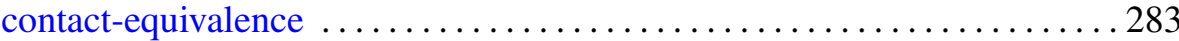

Douglas Martin Grenier, Fundamental domains for the general linear group

Ronald Scott Irving and Brad Shelton, Loewy series and simple projective

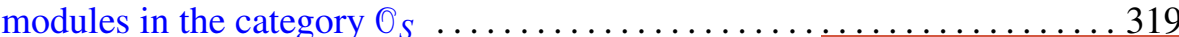

Russell Allan Johnson, On the Sato-Segal-Wilson solutions of the K-dV equation

Thomas Alan Keagy and William F. Ford, Acceleration by subsequence transformations

Min Ho Lee, Mixed cusp forms and holomorphic forms on elliptic varieties

Charles Livingston, Indecomposable surfaces in 4-space 